\title{
Understanding the Effects of Gamification and Juiciness on Players
}

\author{
Kieran Hicks \\ School of Computer Science \\ University of Lincoln \\ Lincoln, Lincolnshire \\ khicks@lincoln.ac.uk \\ Tom Pike \\ School of Life Sciences \\ University of Lincoln \\ Lincoln, Lincolnshire \\ tpike@lincoln.ac.uk
}

\author{
Kathrin Gerling \\ KU Leuven \\ e-Media Research Lab \\ Leuven, Belgium \\ kathrin.gerling@kuleuven.be \\ Oliver Burman \\ School of Life Sciences \\ University of Lincoln \\ Lincoln, Lincolnshire \\ oburman@lincoln.ac.uk
}

\author{
Graham Richardson \\ School of Life Sciences \\ University of Lincoln \\ Lincoln, Lincolnshire \\ grichardson@lincoln.ac.uk \\ Patrick Dickinson \\ School of Computer Science \\ University of Lincoln \\ Lincoln, Lincolnshire \\ pdickinson@lincoln.ac.uk
}

\begin{abstract}
Gamification is widely applied to increase user engagement and motivation, but empirical studies on effectiveness are inconclusive, and often limited to the integration of tangible elements such as leaderboards or badges. In this paper, we report findings from a study with 36 participants that uses the lens of Self-Determination Theory to compare traditional gamification elements, and the concept of juiciness (the provision of abundant audiovisual feedback) in the VR simulation Predator!. Results show that gamification and juiciness improve user experience, but that only juiciness fulfills all basic psychological needs that facilitate intrinsic motivation when applied in nongaming settings. User preferences favour the combination of both approaches, however, neither improved performance, and there is evidence of juicy elements influencing user behaviour. We discuss implications of these findings for the integration of gamification, reflect on the role of both approaches in the context of feedback, and outline challenges and opportunities for further research.
\end{abstract}

Index Terms-Gamification, Game Design, Virtual Reality

\section{INTRODUCTION}

Gamification - the use of game elements in non-gaming settings to increase user engagement and improve performance [8] - is widely applied to transfer the motivational pull of games and increase user engagement with otherwise monotonous tasks [4]. While there is growing empirical evidence of the general effectiveness of gamification [7], [35], many studies only report small effect sizes (e.g., [6]) or omit further statistical analysis [35]. Additionally, our understanding of underlying mechanisms remains limited, with recent large-scale studies returning inconclusive results. For example, Mekler et al. [26] found that the inclusion of badges, levels and leaderboards influenced user performance, but had no significant effect on perceived competence and intrinsic motivation. Taking a slightly different perspective, Sailer et al. [34] included a wider range of game elements and features (e.g., simulated teammates, avatars, and narrative). Results show that aspects such as teammates do not only affect productivity, but also the underlying experience.

An alternative approach toward increased player engagement that has received substantial attention in the game development community (e.g., [18], [39]) is the concept of juiciness, referring to when one player action triggers multiple visual and audio reactions [21]. The concept originated from designers wanting to foster positive game 'feel' [36], and places a focus on immediate additional feedback for player actions which is intended to create an engaging experience. An example of juiciness is the distinct audio that accompanies the player pressing the jump button in Super Mario Bros [28] that supports the visual feedback provided by the game. It has been hypothesized that juiciness can be leveraged to improve player experience [21], suggesting that it might serve as an alternative to gamification for improving user engagement. Yet, no existing empirical work has directly compared elements of juicy design and traditional approaches to gamification (i.e., badges, levels, and leaderboards) when applied in non-gaming settings.

In our work, we study the effects of gamification and juiciness on user experience, behaviour, and performance from the perspective of Self-Determination Theory (SDT) [32]. We use the simple virtual reality (VR) simulation Predator! [31], an application that was originally developed as a research tool to study human ability to track prey exhibiting different fleeing patterns. To understand effects of gamification and explore the impact of juiciness, we created adapted versions of Predator! which include gamification elements, 'juicy' aspects, and a combination of both. Results of a study with 36 participants show that traditional gamification and gamification achieved through juiciness both have positive effects on participants' experience, but that only juiciness offers a significant increase in perceived competence, suggesting that its effect are different from traditional gamification. Likewise, user preferences were 
in favour of the combination of both approaches, however, neither improved user performance.

\section{BACKGROUND}

\section{A. Gamification}

The most widely accepted definition of gamification is provided by Deterding et al. [8], and refers to the "use of design elements characteristic for games in non-game contexts". While this definition in principle covers the application of various characteristics of games, gamification often narrowly focuses on the transfer of a small set of game elements thought to increase user motivation and performance, including points, badges, progression systems, leaderboards, and social comparisons [35]. To explain the effects of gamification on users, an increasing body of research draws from Self-Determination Theory (SDT) [32]: SDT is a psychology-based methodology in-which humans are considered to be naturally intrinsically motivated when their base needs are satisfied, competence, autonomy, and relatedness. Ryan et al [33] apply SDT in the context of games and show that intrinsic motivation is a key factor in encouraging (re-)engagement with games. The theory has been applied in a number of gamification projects. For example, [11], [25] provide quantitative studies that employ SDT as a lens to examine the effects that specific gamification elements have on users, and Deterding [10] explores the relationship between autonomy and experience in a qualitative setting.

Empirical Studies Exploring Effectiveness. A growing body of research explores the effectiveness of gamification. Both Hamari et al. [14] and Seaborn and Fels [34] provide survey papers summarizing evidence of effective application of gamification across a range of settings; for example, education, health, and crowdsourcing, but also criticize methodological weaknesses of many studies. More recently, large-scale studies exploring the effects of gamification have returned inconclusive results. Trying to link 'traditional' gamification elements to theoretical frameworks of motivation, Mekler et al. [26] study the effects of points, levels and leaderboards through the lens of SDT. While the study did find an impact of these gamification elements on performance, the authors did not find a significant increase in perceived competence and intrinsic motivation. In contrast, work by Sailer et al. [34] found a small effect of traditional gamification elements on competence, while non-traditional elements such as simulated teammates positively influenced aspects such as relatedness. Further research by Koivisto and Hamari [23] shows that positive effects of gamification decline over time, outlining an area of attention for future research.

\section{B. Juiciness}

Juiciness refers to large amounts of visual and audio feedback that games can provide to players [12], [15], [21]. For example, Peggle [30] rewards the player with music, ascending tones and, particle effects when the player completes a level, thereby reinforcing the notion that the player is successfully progressing through the game. Game designers have discussed the usefulness of the term [39], while industry postmortems reflect on the implementation and effect of adding juiciness [24]. While Juul's definition of juiciness primarily focuses on positive feedback [20], Swink [36] argues that both negative and positive feedback need to be considered, and draws attention to the immediacy and abundance of feedback as a core aspect contributing to a game being perceived as juicy. Swink hypothesized that juiciness can contribute to perceived player competence and overall player experience, leading to increased player engagement [36]. In contrast to the welldefined list of gamification elements, no clear overview of juicy elements is available. A first attempt at operationalizing juicy design in a similar fashion has been undertaken by Hicks et al. [15], drawing from a systematic analysis of interviews with game developers to de-construct elements of juiciness: they suggest that the concept relates to coherence of game elements, unobtrusive communication of the game state, and the provision of appropriate and direct feedback.

Empirical Studies Exploring Effectiveness. There is little empirical work exploring the effects of juiciness. A 2016 study by Juul and Begy [21] with 46 participants did not reveal significant differences between the juicy and non-juicy version of a tile matching game, but also did not employ standardized measures of player experience or motivation. However, related work has demonstrated an effect of graphical fidelity on player experience [13], and there is evidence that visual appeal positively influences task success rate in serious games [38]. Tapping into gamification and juiciness, Berengueres et al. [2] show that recycling bins equipped with screens to display emoticons that provide immediate feedback increase recycling rates, and were preferred by users. This suggests that juiciness has potential to engage users particularly in nongaming settings, an aspect that we leverage here.

\section{Integration of Gamification and Juiciness in VR}

There is little work that has explored the application of juiciness to VR. Relevant work studying how gamification can be leveraged to increase engagement with VR simulations strongly focuses on healthcare settings; for example, to treat arachnophobia [27], and to train users in the use of hearing aids [29]. Results suggest that gamified VR simulations are an effective means of providing therapy and to engage users. However, the studies did not differentiate between traditional VR simulations and gamified versions, therefore not providing insights into added benefits of gamification and confounding factors such as the novelty of the VR experience.

\section{Feedback in games}

Jarvinen explored the different visual styles present in games and how these audiovisual elements work breaking down the styles into different categories such as soundscape and visual outlook [17]. Anderson and Casey highlight the importance sounds in establishing immersive virtual worlds [1]. Jørgensen also found that players have a large reliance on audio feedback cues in order to effectively play games [19]. While audio has received attention through empirical studies, 
visual feedback elements remain largely understudied in their effect on PX.

In our work, we address the gap in wider perspectives on gamification through implementation of juicy elements, along with those of traditional gamification, allowing us to study their effects on user performance, behaviour, motivation, and experience. This is motivated by the observation that juicy elements may be better aligned with intrinsic motivators, and thus complement traditional approaches toward gamification.

\section{PREDATOR!: A VR SYSTEM TO STUDY GAMIFICATION AND JUICINESS}

The VR simulation Predator! is a system used by researchers in animal behaviour at the University of Lincoln to investigate the efficacy of different real-world prey escape behaviours [31], and has previously been applied in experiments in this field. Given the relatively simple nature of the tool, it offers an ideal test bed for further research. Predator! usage of VR is motivated through its application as a life science tool for exploring fleeing behaviour. Here, we describe the design of the simulation, and how gamification and juicy elements were integrated to complement the existing system.

\section{A. Original Design}

Predator! simulates the task of targeting a moving prey animal in a 3D simulation, which is presented to participants using a Samsung Gear VR Head Mounted Display (HMD). The system uses a simulated prey object, and the targeting process is implemented in a way that is analogous to aligning the prey with the predator's head or body prior to attack (or similar to targeting the prey with a weapon, in the case of a human predator).

1) User Input: Participants undertake a number of trials, each lasting several seconds: they are asked to target (as best as possible) a moving sphere, which represents the prey. Targeting is achieved using a reticle in the centre of the display, and participants need to move their head to align the reticle with the prey as accurately as they can, while it is moving. The prey changes direction and speed (sometimes with high frequency), to confound the targeting process. The ability of the target to evade predation is evaluated using various metrics computed over the course of each trial. Participants perform the task while seated, and receive training in use of the equipment, and in targeting the object, prior to undertaking the experimental conditions.

2) Fleeing Behaviour: The simulation parameters used for our work replicate a study previously performed by Richardson et al [31]. The prey sphere is drawn using a dark colour on a white background to maximise contrast (see Figure 1). Two fleeing behaviours are compared: a "fixed" fleeing behaviour, and a "Protean" behaviour which mimics the movements of certain species [16]. Using the fixed pattern, the prey sphere uses fixed values of turn angle, speed, and frequency of direction change, to define its movement. Protean behaviour uses randomised values, which create more variation and typically confuse predators which try to anticipate prey behaviour. Both fleeing patterns are applied in random order.

3) Feedback Provision: The original simulation includes basic feedback that informs users about the state of the world. Important events (e.g., acquiring the prey) are underlined using simple visual highlights (i.e., briefly changing colour to highlight event); performance feedback is provided implicitly by visualizing the position of the reticle relative to the prey.

\section{B. Gamification Elements}

Gamification has previously been leveraged to increase engagement in a variety of settings, inspired by this work and based on gamification literature [35], we selected four commonly used gamification elements, a scoring system, badges, leaderboards, and progression (see Figure 1).

- The scoring system includes the display of a high score that increments when the user's gaze meets the prey, along with a multiplier for maintained contact.

- Badges are awarded for a number of achievements, for example, completion of five tasks, or maintaining gaze contact with the prey for a certain number of seconds.

- Users are presented with a leaderboard that displays their score in relation to other, simulated users. High scores are adapted to situate the user between rank three and five to minimize effects on player experience [3].

- The simulation includes a progression system based on levels; users are awarded points that translate into levels as they progress through the simulation.

\section{Juicy Elements}

Drawing from previous work on juiciness [15], [21], we selected four elements of juicy design that support the idea of continuous and abundant audiovisual feedback (see Figure 2 for visual feedback elements):

- An animation effect was added to the prey so that it continuously reacts when reached by the user's gaze.

- A particle effect appears when the user initially places their gaze on the prey. The effect spawns around the prey to avoid occlusion of critical information.

- A dynamic soundtrack that is upbeat and pleasant plays with the volume fading based on proximity of the user's gaze to the prey. If they are gazing directly it is full volume, if they are further than the radius of the prey away the music can not be heard.

- A sound effect is played when the user initially places their gaze on the prey. The pitch changes if users rapidly lose and re-gain control of the prey.

Using these elements, we created four versions of Predator!: the original simulation with basic feedback, a gamified version, a juicy version, and a combined version including gamification and juicy elements.

\section{STUDY: UNDERSTANDING THE EFFECTS OF GAMIFICATION AND JUICINESS}

Here we examine the effects of gamification and juiciness using the simulation Predator!. In a within-subjects study 

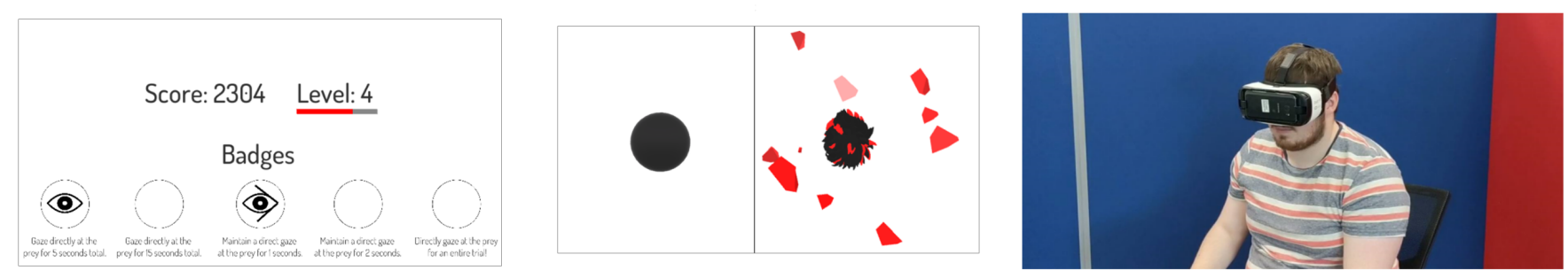

Fig. 1. Left: Presentation of the gamifcation elements. Middle: Basic and Juicy prey including an animation and initial particle effect. Right: A user using the system whilst sitting down in the study enviroment
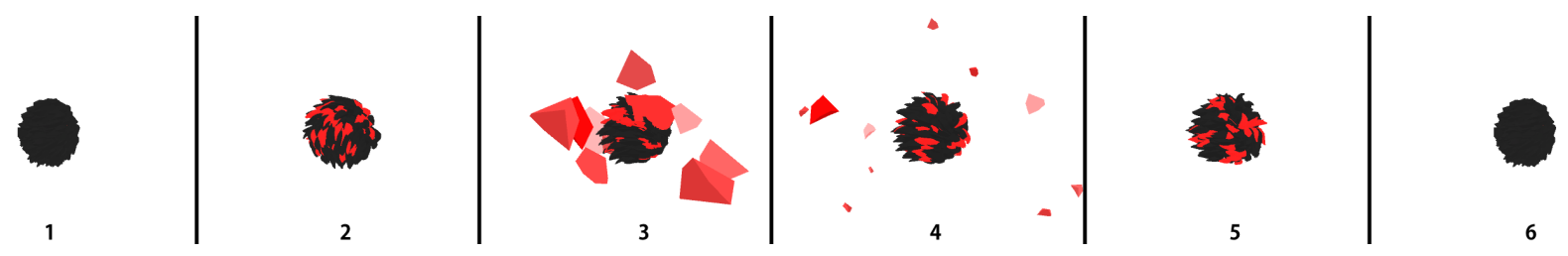

Fig. 2. A visual break down of the juicy visual embellishments that are displayed when the player establishes gaze over the prey in sequential order

with four conditions, we explore how gamified, juicy, and a version including gamification and juicy elements (Combined condition) compare to the standard version of the simulation with regular feedback (Base condition).

\section{A. Research Questions}

Through our work, we aim to address three research questions investigating the effects of gamification and juiciness, and how the approaches are perceived by users.

RQ1: Do gamification and juiciness have an impact on user experience and motivation? Previous work has provided evidence that gamification is an effective means of improving user experience, and literature on juiciness outlines its potential to influence intrinsic motivation through increased competence. This led to the following hypotheses:

Hla: Gamification and juiciness improve user experience.

HIb: Juiciness improves perceived competence and increases intrinsic motivation.

RQ2: Do gamification and juiciness influence user performance and behaviour? Both approaches incorporate elements to encourage user engagement with the core task, and offer additional feedback on performance. However, these amendments to the base version of the simulation could also influence how users act. For example, badges might encourage participants to focus on the accomplishment of related tasks, whereas the nature of juicy design might encourage behaviours that trigger feedback, e.g., re-acquiring the prey.

H2a: Gamification and juiciness increase user performance.

H2b: Gamification will lead to improved metrics that are reflected through badges (maximum time gazed at prey).

H2c: Juiciness will lead to increased participant attempts to trigger feedback (re-acquisition of the prey).

RQ3: Are there differences in the objective effects and perceived benefits of juiciness and gamification? Elements of gamification and juiciness are visible to users and change the appearance of the system to resemble that of a game, possibly increasing its appeal. We therefore hypothesize: H3: Gamification and juiciness improve user perspectives on the simulation; the combination yields best results.

\section{B. Measures}

1) Questionnaires: We employed two standardized questionnaires, the Player Experience and Needs Satisfaction (PENS) questionnaire and the Intrinsic Motivation Inventory (IMI) [5]. The PENS is based on Self-Determination Theory [32], [33] and includes sub-scales for Competence, Autonomy, Presence, Relatedness, and Intuitive Controls. Participants are asked to rate statements such as "I feel competent at the game" on a 7-point Likert scale. The IMI focuses on intrinsic motivation; the version included in this study features three subscales, Interest/Enjoyment, Competence, and Tension. Participants are asked to rate statements such as "I felt pretty skilled at this task" on a 7-point Likert scale. Both questionnaires have previously been applied in Games User Research and studies focusing on gamification (e.g, [26], [34]), demonstrating their suitability in interactive settings. Additionally, we employed an exit questionnaire that asked participants to rank conditions in order of preference, rate the enjoyment of each condition on a 7-point scale, and comment on their preferences.

2) Performance Metrics: The simulation Predator! was originally developed to monitor how well humans can track fleeing behaviors of prey. The original simulation operationalized performance through the average distance of the user's gaze from the prey. Distance is calculated from the Cartesian coordinates of the prey in 3D space, and the orientation of the player's head, recorded every 0.02 seconds. The minimum $3 \mathrm{D}$ distance between the two is calculated using a ray cast from the head of the player to the center of the prey. We adopt this metric as our key measure of performance, along with the score (only displayed in gamified conditions, but recorded for all). Furthermore, we record the longest duration the participant held their gaze directly on the prey as an 
aspect of player behaviour that directly relates to gamification elements (e.g., badges rewarding gaze duration). We include the number of times the prey was acquired by directly gazing at it as a measure of the impact of juicy design on behavior as the acquisition of the prey results in audiovisual feedback.

\section{Participants and Procedure}

We recruited 36 participants (17 male, average age 26, $\mathrm{SD}=5.8)$ through mailing lists and social media sites. Nine Participants had no previous experience using VR, and none of the participants reported colour vision deficiency. Each session lasted about 45 minutes. At the start of the study, each participant provided informed consent and was briefed that they will be playing a prey catching game and how it is played. Afterwards, participants were given brief background information on Predator! as a research tool for the life sciences. When participants first put on the VR HMD, they were shown instructions to help focus and ensure the headset was comfortable, and they were also given the chance to practice on a trial task until they felt ready to proceed whilst being guided by the investigator. The remainder of the study was split into four sequences consisting of one of the conditions (Base, Juicy, Gamified, Combined) of Predator! followed by questionnaires on player experience and motivation. Conditions were counterbalanced using a Latin square to control for order effects. At the end of the study, participants were asked to complete a final questionnaire on their experience, and to provide demographic information. Afterwards, participants were given an opportunity to ask questions relating to the conditions and research. The research was approved by the ethics board at the University of Lincoln. All participants gave informed consent to the use of their responses for analysis and publication. As part of our health and safety routine, participants were asked to immediately report simulator sickness, and answered the Simulator Sickness Questionnaire (SSQ) [22] at the end of the study. Results show no instances of sickness.

\section{Data Analysis}

Data were analyzed in SPSS. We applied RM-ANOVAs for questionnaire data (PENS and IMI) and performance data using condition as within-subjects factor. If sphericity was violated, we applied Huynh-Feldt correction; pairwise comparisons were made with Bonferroni correction. Preferences were analyzed using Friedman's Analysis of Variance, pairwise comparisons were made using Wilcoxon Signed Rank tests.

\section{E. Results}

Here, we present our results organized by research questions. We report quantitative results and further explain our findings using qualitative participant feedback.

1) RQ1: Do Gamification and Juiciness have an impact on user experience and motivation?: Yes. Our results show that the Juicy, Gamified and Combined conditions of the study provided a significantly better user experience than the Base version of the simulation, and also resulted in significantly higher levels of user motivation (see Table 1 for descriptives).
Player experience. There was a main effect of condition on Competence $\left(F_{3,105}=4.618, p=.004, \eta^{2}=.117\right)$. Pairwise comparisons showed that juicy elements increased competence $(p=.002)$, but that Gamification $(p=.723)$ and the Combined version $(p=.336)$ did not contribute to participants' perception of competence when compared to the Base version. We also found a main effect of condition on Autonomy $\left(F_{3,105}=8.475, p=.000, \eta^{2}=.195\right)$. Pairwise comparisons showed that the Juicy $(p=.005)$, Gamified $(p=.000)$ and Combined versions $(p=.002)$ all significantly contributed to perceived autonomy, but none of these versions outperformed each other (all $p=1.000$ ). Further, there was a main effect of condition on Relatedness $\left(F_{3,105}=4.258\right.$, $\left.p=.007, \eta^{2}=.108\right)$. Pairwise comparisons showed that the Juicy $(p=.018)$ and Combined versions $(p=.023)$ significantly improved Relatedness, but that there was no difference between them $(p=1.000)$. We also found a main effect of condition on Presence $\left(F_{3,105}=8.215, p=.000, \eta^{2}=.190\right)$. Pairwise comparisons showed that the Juicy $(p=.000)$, Gamified $(p=.000)$ and Combined versions $(p=.003)$ all significantly increased presence, but none of these versions outperformed each other (all $p=1.000$ ). Finally, there was no significant main effect of condition on Intuitive Controls $\left(F_{2.44,85.60}=2.262, p=.099, \eta^{2}=.061\right)$, suggesting that the control scheme was perceived as comparable across conditions. These results support H1a.

Intrinsic motivation. We found a main effect of condition on Interest/Enjoyment $\left(F_{3,105}=13.076, p=.000, \eta^{2}=\right.$ $.272)$. Pairwise comparisons showed that the Juicy $(p=.000)$, Gamified $(p=.001)$ and Combined versions $(p=.000)$ all significantly increased interest/enjoyment, but there were no significant differences between them (all $p=1.000$ ). Further, there was a main effect of condition on Competence $\left(F_{3,105}=6.739, p=.000, \eta^{2}=.161\right)$. Pairwise comparisons showed that juicy elements increased competence $(p=.000)$, but that Gamification $(p=.259)$ and the Combined version $(p=.379)$ had no effect when compared to the base version. This result supports H1b. Finally, there was no significant main effect of condition on Choice $\left(F_{3,105}=.028, p=.993\right.$, $\left.\eta^{2}=.001\right)$ and Tension $\left(F_{2.40,84.09}=.892, p=.430\right.$, $\left.\eta^{2}=.025\right)$.

2) RQ2: Do Gamification and Juiciness influence user performance and behaviour?: Only in some instances. Our results show that there is no effect of Gamification, Juiciness or the Combined version on the original performance metric. However, our results do show that the maximum time spent gazing directly at the prey was lowest in the Juicy and Combined conditions, suggesting that Juicy elements can have an impact on player behaviour that in turn affects performance metrics. We found no significant main effect of condition on average distance from the prey $\left(F_{2.4,84.24}=.107, p=.928\right.$, $\eta^{2}=.003 ; M_{\text {Base }}=0.46, S D_{\text {Base }}=0.66, M_{\text {Juicy }}=0.48$, $S D_{\text {Juicy }}=0.66, M_{\text {Gamified }}=0.48, S D_{\text {Gamified }}=0.77$, $M_{\text {Combined }}=0.49, S D_{\text {Combined }}=0.83$ ), the performance metric applied in the original animal behaviour study. Therefore, $\mathrm{H} 2 \mathrm{a}$ cannot be confirmed. However, we did find a main effect 
TABLE I

AVERAGE SCORES FOR THE PENS AND IMI (7-POINT LIKERT SCALE) FOR EACH CONDITION.

\begin{tabular}{llllllllll} 
& & \multicolumn{2}{c}{ Base } & \multicolumn{2}{c}{ Juicy } & \multicolumn{2}{c}{ Game } & \multicolumn{2}{c}{ Both } \\
\hline \multirow{3}{*}{ PENS } & & M & SD & M & SD & M & SD & M & SD \\
& Competence & 3.84 & 1.71 & 4.84 & 1.57 & 4.24 & 1.71 & 4.40 & 1.51 \\
& Autonomy & 3.04 & 1.36 & 3.89 & 1.39 & 4.11 & 1.45 & 4.09 & 1.57 \\
& Relatedness & 1.89 & 1.05 & 2.42 & 1.33 & 2.30 & 1.20 & 2.44 & 1.30 \\
& Presence & 2.29 & .83 & 3.04 & .87 & 2.9 & 1.01 & 3.0 & 1.04 \\
& Intuitive controls & 6.4 & .83 & 6.56 & .56 & 6.55 & .77 & 6.70 & .50 \\
\hline IMI & Interest / enjoyment & 3.86 & 1.33 & 4.94 & 1.20 & 4.74 & 1.23 & 5.03 & 1.18 \\
& Perceived competence & 3.52 & 1.55 & 4.61 & 1.44 & 4.08 & 1.44 & 4.12 & 1.44 \\
& Perceived choice & 5.85 & 1.07 & 5.87 & .84 & 5.83 & 1.01 & 5.87 & 1.12 \\
& Pressure / tension & 2.61 & 1.21 & 2.29 & .99 & 2.47 & 1.30 & 2.50 & 1.20 \\
\hline
\end{tabular}

of condition on the maximum time that participants spent holding a direct gaze on the prey $\left(F_{2.373,83}=3.4, p=.032\right.$, $\left.\eta^{2}=.088\right)$. Pairwise comparisons showed that the Juicy condition significantly decreased time spent over prey when compared to the Base $(p=.044)$ and Gamification $(p=.003)$. In the Combined version, participants also spent significantly less time directly gazing at the prey when compared against the Base version $(p=.034)$. This directly contradicts $\mathrm{H} 2 \mathrm{~b}$ suggesting that the Gamified condition increases maximum time spent gazing at the prey; instead, the inclusion of juicy elements reduces the time spent gazing at the prey compared to the other conditions. This is also reflected in the score calculated based on these values; there was a significant main effect of condition on score $\left(F_{3,105}=3.964, p=.010\right.$, $\eta^{2}=.102 ; M_{\text {Base }}=9673, S D_{\text {Base }}=4036, M_{\text {Juicy }}=9006$, $S D_{\text {Juicy }}=3161, M_{\text {Gamified }}=10559, S D_{\text {Gamified }}=4525$, $M_{\text {Combined }}=9346, S D_{\text {Combined }}=3269$ ). Pairwise comparisons show no significant difference was between Base and Juicy $(p=.196)$, Gamification and Base $(p=.077)$, and Combined version and Base $(p=.442)$. However, participants scored significantly higher in the Gamified condition when compared to both the Juicy $(p=.001)$ and Combined $(p=.019)$ versions. Finally, we did not find significant differences regarding the amount of times users re-acquired prey $\left(F_{2.41,84.45}=.986\right.$, $\left.p=.390, \eta^{2}=.027\right)$. Thus, H2c cannot be supported.

3) RQ3: Are there differences in the objective effects and perceived benefits of juiciness and Gamification?: Yes. Results for perceived enjoyment and overall preference of condition (version of the simulation) suggest that subjective preference was highest for the Combined version featuring both juicy and gamification elements, and that the Juicy version was preferred to the Gamified version. However, qualitative feedback does not just highlight benefits of juiciness but also suggests that participants appreciated traditional gamification.

Perceived enjoyment. We found a main effect of condition on perceived enjoyment $\left(F_{2.72,95.29}=53, p=.000, \eta^{2}=\right.$ .602). Pairwise comparisons revealed that all conditions were rated significantly different from each other. The Combined version was rated significantly higher than Base $(p=.000)$, Juicy $(p=.001)$ and, Gamification $(p=.000)$. Further, the Juicy version was rated significantly higher than both the Base $(p=.000)$ and, Gamification $(p=.000)$ versions. Lastly, Gamification was rated significantly higher than the Base $(p=$
TABLE II

AVERAGE RATINGS AND SD FOR ENJOYMENT ( $1=$ NOT AT ALL, $7=$ VERY MUCH) AND CONDITION PREFERENCE RANKINGS (MEDIAN VALUE).

\begin{tabular}{lcc} 
& Enjoyment & Ranking \\
\hline Base & $2.81(1.61)$ & 4.00 \\
\hline Juicy & $5.58(1.13)$ & 2.00 \\
\hline Gamification & $4.64(1.55)$ & 3.00 \\
\hline Combined & $6.28(0.77)$ & 1.00 \\
\hline
\end{tabular}

.000) version. These results support $H 3$.

Preference. We found a main effect of condition on preference ranking order $\left(\chi^{2}(2)=67.9, p=.000\right)$. Pairwise comparisons revealed that all conditions were ranked significantly different from each other. The Combined version was rated significantly higher than all three conditions (Base $Z=-5.34$, $p=.000$, Juicy $Z=-3.26, p=.001$, Gamified $Z=-4.66$, $p=.000)$. The Juicy condition was rated significantly higher than both Base $(Z=-5.21, p=.000)$ and Gamified $(Z=-2.45, p=.014)$ conditions. Lastly, Gamification was rated significantly higher than Base $(Z=-3.75, p=.000)$. These results also support $H 3$.

Finally, qualitative feedback further elaborates on participant preferences and ratings. Regarding juicy design, participants reported that associated elements increased feelings of engagement. For example, one participant stated that "I liked the music it made the game a lot more exciting and engaging." Some participants also commented on how juicy elements made the prey more relatable, e.g., outlining that "I appreciated the furry ball it felt more alive". When commenting on Gamification elements, participants reported enjoying the goal orientated nature that badges provided, e.g., "Getting to see what badges you can get to challenge myself." Gamification elements were also found to be motivating, e.g., one participant stated that "The score and badges encouraged me to play more." Further, feedback that the elements provided was also observed by participants: "The feedback supplied helped gain an understanding of what I was doing." Finally, participants enjoyed the combination of the elements as it helped foster feelings of engagement, for example, "The combined elements made the game more engaging and enjoyable." 


\section{Discussion}

Our work examines the effects of traditional gamification and gamification implemented by means of juicy design. Here, we discuss the implications of our findings with a focus on differences between gamification and juiciness, and challenges and opportunities regarding their implementation.

\section{A. Effects of Gamification and Juicy Design in Simple VR}

Our results show that traditional gamification and juicy design both offer effective means of improving user experience particularly when asking users to engage with otherwise simplistic but challenging task in a VR setting.

1) Effects on User Performance and Behaviour: Neither gamification nor juiciness led to significant increases in performance. This result needs to be interpreted in the light of the given task and the fidelity of the environment: tracking fleeing behavior of a virtual object, and asking individuals to operate at the fringes of their abilities, possibly leaving little room for improvement. Additionally, our results show that juiciness affected participant behaviour (shorter maximum time that gaze was held directly on the prey), whereas gamification had no impact. We hypothesize that this difference may be a result of additional visual feedback that is displayed around the prey and on its acquisition in conditions with juicy elements, possibly introducing a source of distraction. This aspect highlights a core challenge in the employment of juiciness: elements need to be chosen in a way that they do not act as a confound. For example, if a task is predominantly visual, graphical effects can be problematic, and alternatives such as audio feedback need to be considered.

2) User Preferences: Gamification vs Game: Beyond the direct effects of both approaches, our findings reveal that the Combined (gamified and juicy) version received the highest preference ratings. This hints at an interesting dilemma: our combined version arguably did not just integrate two approaches toward the transfer of game elements into nongaming settings, but also most closely resembled an actual game due to the number of game elements that were integrated. Researchers and designers wishing to employ gamification and related approaches therefore need to answer two questions: What is the minimum number of game elements required to meet the threshold for positive user feedback in a given scenario, and when does a gamified system become a game a question that becomes increasingly relevant as the boundaries between gamified systems and games shift.

3) Impact of the VR Environment: Finally, it is important to consider the impact of the VR environment. For our study, we worked with a simulation environment designed for life sciences research; the simplicity of the environment clearly exposed elements of gamification and juicy design, and in applications that are more complex (both in terms of tasks and visual design) achieving an impact of juicy design might be more challenging. To address this issue, future work should explore how juicy design compares to gamification both in more complex VR environments, but also in non-VR settings.

\section{B. Gamification, Juiciness, and Self-Determination Theory}

Previous work suggests a relationship between gamification and intrinsic motivation that requires careful selection of game elements, with some studies suggesting it neither has positive nor detrimental effects [26], and others showing that a broad range of elements (e.g., badges, leaderboards and social avatars) should be applied to achieve improvements in all aspects (competence, autonomy, relatedness) [34]. In this context, our results demonstrate that simple juicy elements have clear benefits for perceived competence, autonomy, and relatedness (in turn increasing intrinsic motivation) in the given setting, suggesting that this approach may be leveraged as a design alternative to traditional gamification. This is in line with previous research on SDT [5], demonstrating that tangible rewards decrease intrinsic motivation, whereas feedback that emphasizes competence while maintaining autonomy has a positive impact on intrinsic motivation.

\section{Back to the Roots of Gamification}

Our findings suggest that we need to reconsider the pervasive perspective on gamification that exclusively focuses on elements such as badges, levels, and leaderboards. We bring the concept back to the original definition that considered any application of game elements in a non-gaming context to be gamification [8], and responds to recent criticisms that call for a focus on gameful experiences [9]. Our results offer an opportunity for researchers and designers wishing to apply gamification: While 'traditional' (i.e., commonly applied) gamification elements focus on performance (e.g., through leaderboards), elements of juicy design leverage realtime feedback to inform users about achievement and make them feel more connected with the system with both approaches effectively complementing each other. We therefore suggest explicitly incorporating juiciness in the definition of gamification as an approach that leverages the application of game elements in non-gaming settings. Therefore, gamification encompasses performance-centred aspects (traditional gamification elements such as leaderboards and levels), but also includes experience-centred design elements (juicy elements including immediate audiovisual feedback).

\section{LIMITATIONS AND FUTURE WORK}

There are some limitations that need to be considered when interpreting our work. First, our study was carried out using a simplistic VR simulation; while it offered an ideal test bed for an initial study, further research is necessary to extend our findings to other settings (e.g., desktop applications or mobile apps). Additionally, studies involving more complex tasks and implementing different VR systems are necessary to ensure that findings can be generalised. In the future, we also plan to study the impact of separate elements (e.g., audio feedback compared to visual feedback) to gain further insights into the effects that juicy design may have. Finally, results reported in this paper were obtained through a single-session lab study. To further explore the motivational pull of gamification and juiciness, we believe that the long-term study of its impact 
on users is necessary, spanning a bigger number of research sessions, and including an in-the-wild deployment that leaves more room for player-initiated engagement.

\section{CONCLUSION}

In this paper, we explored the effects of gamification and juiciness. Our results show that both gamification and juiciness can be effective means of delivering a positive user experience, but that in the context of our study, only juiciness significantly improves the three basic psychological needs for competence, autonomy and relatedness, in turn facilitating intrinsic motivation. These findings have implications for our perspectives on gamification, suggesting that designers should refocus on the development of a wider, experience-centred toolbox that move beyond the application of traditional gamification elements (as provided by [9]), and equips researchers and designers with broader means of creating engaging playful experiences.

\section{REFERENCES}

[1]

[2] J. Berengueres, F. Alsuwairi, N. Zaki, and T. Ng, "Gamification of a recycle bin with emoticons," in Proceedings of the 8th ACM/IEEE international conference on Human-robot interaction. IEEE Press, 2013, pp. 83-84.

[3] M. V. Birk, R. L. Mandryk, M. K. Miller, and K. M. Gerling, "How selfesteem shapes our interactions with play technologies," in Proceedings of the 2015 Annual Symposium on Computer-Human Interaction in Play. ACM, 2015, pp. 35-45.

[4] M. V. Birk, R. L. Mandryk, and C. Atkins, "The motivational push of games: The interplay of intrinsic motivation and external rewards in games for training," in Proceedings of the 2016 Annual Symposium on Computer-Human Interaction in Play. ACM, 2016, pp. 291-303.

[5] E. Deci and R. M. Ryan, Intrinsic motivation and self-determination in human behavior. Springer Science \& Business Media, 1985.

[6] P. Denny, "The effect of virtual achievements on student engagement," in Proceedings of the SIGCHI conference on human factors in computing systems. ACM, 2013, pp. 763-772.

[7] P. Denny, F. McDonald, R. Empson, P. Kelly, and A. Petersen, "Empirical support for a causal relationship between gamification and learning outcomes," in Proceedings of the 2018 CHI Conference on Human Factors in Computing Systems. ACM, 2018, p. 311.

[8] S. Deterding, D. Dixon, R. Khaled, and L. Nacke, "From game design elements to gamefulness: defining gamification," in Proceedings of the 15th international academic MindTrek conference: Envisioning future media environments. ACM, 2011, pp. 9-15.

[9] S. Deterding, "The lens of intrinsic skill atoms: A method for gameful design," Human-Computer Interaction, vol. 30, no. 3-4, 2015.

[10] S. Deterding, "Contextual autonomy support in video game play: a grounded theory," in Proceedings of the 2016 CHI Conference on Human Factors in Computing Systems. ACM, 2016, pp. 3931-3943.

[11] S. F. Forde, E. D. Mekler, and K. Opwis, "Informational vs. controlling gamification: A study design," in Proceedings of the 2015 annual symposium on computer-human interaction in play. ACM, 2015, pp. $517-522$.

[12] K. Gabler, K. Gray, M. Kucic, and S. Shodhan, "enHow to Prototype a Game in Under 7 Days." [Online]. Available: https://www.gamasutra.com/view/feature/130848

[13] K. M. Gerling, M. Birk, R. L. Mandryk, and A. Doucette, "The effects of graphical fidelity on player experience," in Proceedings of international conference on Making Sense of Converging Media. ACM, 2013.

[14] J. Hamari, J. Koivisto, and H. Sarsa, "Does gamification work? - a literature review of empirical studies on gamification," in 2014 47th Hawaii International Conference on System Sciences(HICSS), vol. 00, Jan. 2014, pp. 3025-3034. [Online]. Available: doi.ieeecomputersociety.org/10.1109/HICSS.2014.377

[15] K. Hicks, P. Dickinson, J. Holopainen, and K. Gerling, "enGood game feel: An empirically grounded framework for juicy design," in enProceedings of the 2018 DiGRA Conference, 2018, p. 17.
[16] D. Humphries and P. Driver, "Protean defence by prey animals," Oecologia, vol. 5, no. 4, pp. 285-302, 1970.

[17] A. Järvinen, "Gran Stylissimo: The Audiovisual Elements and Styles in Computer and Video Games," in CGDC Conference. 2002.

[18] M. Jonasson and P. Purho, "Juice It or Lose It." [Online]. Available: https://www.gdcvault.com/play/1016487/Juice-It-or-Lose

[19] K. Jørgensen, "Left in the dark: playing computer games with the sound turned off," in Ashgate. 2008.

[20] J. Juul, A casual revolution: Reinventing video games and their players. MIT press, 2010.

[21] J. Juul and J. S. Begy, "Good feedback for bad players? a preliminary study of juicyinterface feedback," in Proceedings of first joint FDG/DiGRA Conference, Dundee, 2016.

[22] R. S. Kennedy, N. E. Lane, K. S. Berbaum, and M. G. Lilienthal, "Simulator sickness questionnaire: An enhanced method for quantifying simulator sickness," The international journal of aviation psychology, vol. 3, no. 3, pp. 203-220, 1993

[23] J. Koivisto and J. Hamari, "Demographic differences in perceived benefits from gamification," Computers in Human Behavior, vol. 35 , pp. 179-188, 2014.

[24] L. LeRay, "enFilling Luna 's touchable VR gameworld with 'juicy' feedback." [Online]. Available: gamasutra/view/news/285203

[25] E. D. Mekler, F. Brühlmann, K. Opwis, and A. N. Tuch, "Do points, levels and leaderboards harm intrinsic motivation?: an empirical analysis of common gamification elements," in Proceedings of the First International Conference on gameful design, research, and applications. ACM, 2013, pp. 66-73.

[26] E. D. Mekler, F. Brühlmann, A. N. Tuch, and K. Opwis, "Towards understanding the effects of individual gamification elements on intrinsic motivation and performance," Computers in Human Behavior, vol. 71, pp. 525-534, 2017.

[27] A. Miloff, P. Lindner, W. Hamilton, L. Reuterskiöld, G. Andersson, and P. Carlbring, "Single-session gamified virtual reality exposure therapy for spider phobia vs. traditional exposure therapy: study protocol for a randomized controlled non-inferiority trial," Trials, vol. 17, no. 1, 2016.

[28] E. Nintendo, "Super mario bros," Game [NES].(13 September 1985). Nintendo, Kyoto, Japan, 1985.

[29] H. Patel, S. Cobb, M. Hallewell, M. D'Cruz, R. Eastgate, L. Picinali, and S. Tamascelli, "User involvement in design and application of virtual reality gamification to facilitate the use of hearing aids," in Interactive Technologies and Games (iTAG), 2016 International Conference on. IEEE, 2016, pp. 77-81.

[30] PopCap Games, "Peggle," Game [PC], 2007.

[31] G. Richardson, P. Dickinson, O. H. P. Burman, and T. W. Pike, "Unpredictable movement as an anti-predator strategy," Proceedings of the Royal Society of London B: Biological Sciences, vol. 285, no. 1885, 2018. [Online]. Available: http://rspb.royalsocietypublishing.org/content/285/1885/20181112

[32] R. M. Ryan and E. L. Deci, "Self-determination theory and the facilitation of intrinsic motivation, social development, and well-being." American psychologist, vol. 55, no. 1, p. 68, 2000.

[33] R. M. Ryan, C. S. Rigby, and A. Przybylski, "The motivational pull of video games: A self-determination theory approach," Motivation and emotion, vol. 30, no. 4, pp. 344-360, 2006.

[34] M. Sailer, J. U. Hense, S. K. Mayr, and H. Mandl, "How gamification motivates: An experimental study of the effects of specific game design elements on psychological need satisfaction," Computers in Human Behavior, vol. 69, pp. 371-380, 2017.

[35] K. Seaborn and D. I. Fels, "Gamification in theory and action: A survey," International Journal of human-computer studies, vol. 74, pp. 14-31, 2015.

[36] S. Swink, Game feel: a game designer's guide to virtual sensation. CRC Press, 2008

[37] C. Tan, W. Leong, S. Shen, C. Dubravs, C. Si, "Exploring gameplay experiences on the oculus rift," in Proceedings of the 2015 Annual Symposium on Computer-Human Interaction in Play. pp. 253-263, 2015.

[38] V. Vanden Abeele, J. Wouters, P. Ghesquière, A. Goeleven, and L. Geurts, "Game-based assessment of psycho-acoustic thresholds: Not all games are equal!" in Proceedings of the 2015 Annual Symposium on Computer-Human Interaction in Play. ACM, 2015, pp. 331-341.

[39] J. Whitkin, “: a useful game design term?" 2014. [Online]. Available: https://www.gamasutra.com/blogs/JoshWhitkin/ 20140131/209273/Juicy au seful ame $_{d}$ esign $_{t}$ erm.php 